\title{
Porcine Skin as a Source of Biodegradable Matrices: Alkaline Treatment and Glutaraldehyde Crosslinking
}

\author{
Fabiana T. Rodrigues, Virginia C. A. Martins, Ana M. G. Plepis \\ Instituto de Química de São Carlos, USP
}

\begin{abstract}
In this work, the modifications promoted by alkaline hydrolysis and glutaraldehyde (GA) crosslinking on type I collagen found in porcine skin have been studied. Collagen matrices were obtained from the alkaline hydrolysis of porcine skin, with subsequent GA crosslinking in different concentrations and reaction times. The elastin content determination showed that independent of the treatment, elastin was present in the matrices. Results obtained from in vitro trypsin degradation indicated that with the increase of GA concentration and reaction time, the degradation rate decreased. From thermogravimetry and differential scanning calorimetry analysis it can be observed that the collagen in the matrices becomes more resistant to thermal degradation as a consequence of the increasing crosslink degree. Scanning electron microscopy analysis indicated that after the GA crosslinking, collagen fibers become more organized and well-defined. Therefore, the preparations of porcine skin matrices with different degradation rates, which can be used in soft tissue reconstruction, are viable.
\end{abstract}

Keywords: Porcine skin, collagen, glutaraldehyde crosslinking.

\section{Introduction}

Cutaneous lesions, diseases and burns are considered the main factor of soft tissue damage. Apart from the loss of tissue function, such impairment generates social and psychological tribulations. The reconstruction or replacement of damaged tissues is one of the objectives behind tissue engineering, especially in substantial burns and chronic wounds, because current treatments are insufficient in preventing scar formation and promoting restorative healing ${ }^{[1]}$. Moreover, tissue loss can be extensive and the healing process may not be sufficient for a theraupetic healing of the tissue. Thus, the use of grafts should be considered ${ }^{[2,3]}$. Acellular matrices for tissue engineering are currently being prepared to serve as scaffolds for new tissue growth ${ }^{[4]}$. The devitalization of homologous or heterologous matrices is an alternative for the production of dermal matrices ${ }^{[5]}$. Tissues of animal origin have been used for thousands of years to cover extensive wounds. Their unlimited availability is one of the advantages in using heterologous matrices. Porcine skin is one of the several heterologous matrices under study, mainly on account of its high similarity to human skin and its biocompatibility, mechanical resistance and low antigenicity ${ }^{[6]}$. Porcine skin is a well-suited temporary wound dressing for seconddegree burns and usually promotes scar-free healing with an average healing time of about 10 days ${ }^{[5]}$.

Collagen matrices can be chemically modified due to their large quantities of reactive groups such as amines $\left(\mathrm{NH}_{2}\right)$, carboxylic acids $(\mathrm{COOH})$ and hydroxyl $(\mathrm{OH})$; allowing the implementation of different structural, mechanic and physico-chemical properties, making possible new applications in the biomaterials field. One possible chemical modification is the selective hydrolysis of carboxyamide groups of asparagine and glutamine residues present in type I collagen ${ }^{[7]}$.

This modification generates a negatively charged matrix at $\mathrm{pH} 7.4$, with a total increase up to $134 \pm 12$ negative charges per tropocollagen unit after 96 hours of hydrolysis ${ }^{[7]}$. Collagen matrices obtained by alkaline hydrolysis of bovine pericardium were highly biocompatible, and in rat tibia experiments, the material incorporated in the tissue with an absence of a chronic inflammatory response ${ }^{[8,9]}$.

Moreover, an important property for collagen matrices is biodegradability, which should occur with the new tissue formation; therefore a matrix degradation rate control is interesting and can be obtained by crosslinking reactions. Collagen is a very promising raw material for tissue engineering, however it is easily biodegradable. Crosslinking of collagen-based matrices is an effective method to modify biodegradation rates and to improve mechanical properties ${ }^{[10-12]}$.

Chemical crosslinking can result in materials which are permanently crosslinked ${ }^{[13]}$, and the in vivo degradation rate of these materials may be controlled ${ }^{[14]}$. Glutaraldehyde (GA) is a crosslinking agent used in collagen matrices that do not affect biocompatibility and increase biological stability ${ }^{[15,16]}$.

The objective of this study was the preparation and characterization of collagen type I matrices submitted to an alkaline hydrolysis and GA crosslinking. Collagen matrices were obtained by alkaline hydrolysis of porcine skin, with subsequent GA crosslinking, in different concentrations $(0-0.1 \%)$ and reaction times (15 and 45 minutes).

Autor para correspondência: Ana M. G. Plepis, Instituto de Química, Universidade de São Paulo, Caixa Postal 780, CEP: 13560-970 São Carlos, SP, Brasil. 


\section{Experimental}

\section{Matrices preparation}

Porcine skin obtained from a slaughterhouse was used as raw material. It was removed from the porcine abdominal wall and underwent mechanical cleaning up. It was then alternately immersed several times in distilled water (30 minutes) and $\mathrm{NaCl} 0.9 \%$ (1 hour), at $25{ }^{\circ} \mathrm{C}$. For the alkaline hydrolysis, porcine skin was prepared as aforementioned for bovine pericardium ${ }^{[17]}$. Briefly, the porcine skin was immersed for 96 hours at $25{ }^{\circ} \mathrm{C}$ in an alkaline solution $\left(3 \mathrm{~mL} . \mathrm{g}^{-1}\right.$ of tissue $)$ containing salts of alkaline and alkaline earth metals. The resulting material was equilibrated in a solution containing salts of alkaline and alkaline earth metals, $\left(6 \mathrm{~mL} \cdot \mathrm{g}^{-1}\right.$ of tissue $)$ for 6 hours. Excess salts were removed by rinses in 3\% (w/w) boric acid solution, deionized water, followed by $0.3 \%(\mathrm{w} / \mathrm{w})$ EDTA solution, $\mathrm{pH} 11$ and finally equilibrated in $0.13 \mathrm{~mol} . \mathrm{L}^{-1}$ phosphate buffer, pH 7.4 (PB). The matrices obtained were denominated as HPS (hydrolyzed porcine skin).

\section{Glutaraldehyde (GA) crosslinking methods}

To improve the biostability, after alkaline hydrolysis, the HPS was crosslinked with GA in concentrations ranging from 0.01 to $0.1 \%$ and in two different reaction times (15 and 45 minutes). Three different methods were used for the crosslinking reaction as described below:

\section{Non-sequential crosslinking - 15 minutes}

HPS was divided in 4 pieces. The first piece was crosslinked with a $0.1 \%$ GA solution (01GA15), the second one with a $0.05 \%$ GA solution (005GA15), the third one with a $0.01 \%$ GA solution (001GA15) and the last one was exposed to PB (0GA), all of them for 15 minutes at $25^{\circ} \mathrm{C}$. Afterward, the matrices were exposed to a glycine: borate buffer (GBB - $2.5 \times 10^{-2}$ mol.L $\mathrm{L}^{-1}$ glycine: $5 \times 10^{-2}$ mol.L $\mathrm{L}^{-1}$ borate, $\mathrm{pH}$ 9.2) for 1 hour, washed with $\mathrm{PB}$ and deionized water, and were frozen and lyophilized in the Edwards Model Freeze Dryer Modulyo (Edwards High Vacuum International, West Sussex, UK). The GBB exposure was necessary to eliminate residual GA.

\section{Non-sequential crosslinking - 45 minutes}

HPS was divided in 3 pieces. The first piece was crosslinked with a $0.085 \%$ GA solution (85GA45), the second one with a $0.05 \%$ GA solution (005GA45) and the third one was exposed to PB (0GA), all of them for 45 minutes at $25^{\circ} \mathrm{C}$. Afterward, the matrices were exposed to GBB for 1 hour, washed with PB and deionized water, and were frozen and lyophilized.

\section{Sequential crosslinking - $\mathbf{4 5}$ minutes}

HPS was sequentially crosslinked in three steps:

- A - $0.010 \%$ GA solution, 15 minutes;

- B - $0.025 \%$ GA solution, 15 minutes;

- C $-0.050 \%$ GA solution, 15 minutes.
After sequential crosslinking, the matrix was exposed to GBB for 1 hour, washed with PB and deionized water, and was frozen and lyophilized, obtaining a matrix with a total concentration of GA of $0.085 \%$ and with a total reaction time of 45 minutes (85GA45S).

\section{Characterization}

\section{Determination of the elastin content}

Insoluble elastin was quantified according to its resistance to hydrolysis during treatment with a diluted hot $\mathrm{NaOH}$ solution, according to Cross, Smith and Carpenter (1973). Lyophilized matrices of about $50 \mathrm{mg}$ were submitted to hydrolysis with $0.1 \mathrm{~mol} . \mathrm{L}^{-1}$ (100 mL. $\mathrm{g}^{-1}$ of sample), at $100^{\circ} \mathrm{C}$ for 50 minutes ${ }^{[18]}$. After that time, the samples were washed with deionized water and centrifuged for 5 minutes at $3000 \mathrm{rpm}$. This process was repeated once more and the insoluble elastin residue was frozen and lyophilized and the dry weight was quantifified and expressed as mg per $\mathrm{g}$ of tissue, which corresponds to the average of three independent determinations.

\section{In vitro trypsin degradation}

In vitro biodegradation test was performed by digestion with trypsin (Merck activity of $3.5 \mathrm{U}^{-\mathrm{mg}^{-1}}$ ). The trypsin solution was prepared in 0.2 mol. $\mathrm{L}^{-1}$ borate buffer ( $\mathrm{pH}$ 7.6) containing $5 \times 10^{-3} \mathrm{~mol} . \mathrm{L}^{-1} \mathrm{CaCl}_{2}$, with a total activity of $0.01 \mathrm{U} \cdot \mathrm{mg}^{-1}$ of the sample. Three discs of $1 \mathrm{~cm}$ diameter of each sample were heated for 5 minutes in a water-bath to achieve collagen denaturation and were then frozen and lyophilized until constant weight. The trypsin solution was added to the samples and incubated at $37{ }^{\circ} \mathrm{C}$ for 2 hours. After this time, samples were heated in a water-bath for 3 minutes, washed with deionized water, frozen, lyophilized and weighed for degradation determination.

The biodegradation degree is defined as the relation between non-degraded mass and initial mass of the samples (Equation 1):

$$
\text { Degradation Percentage }=\frac{M t-M r}{M t} \times 100
$$

where $\mathrm{Mr}$ is the dry weight of matrix after degradation with trypsin and $\mathrm{Mt}$ is the initial dry weight of matrix.

\section{Differential Scanning Calorimetry (DSC)}

Thermal denaturation temperature (Td) was determined by Differential Scanning Calorimetry (DSC-2010, TA Instruments, New Castle, USA). Heating was performed in hermetic aluminum pans in synthetic air flow $(80 \mathrm{~mL} / \mathrm{min})$ at a rate of $10{ }^{\circ} \mathrm{C}$ minutes from $5-120^{\circ} \mathrm{C}$. The sample weight was in the range of 9-10 $\mathrm{mg}$ and $\mathrm{Td}$ was obtained from the inflection point of the DSC curves.

\section{Thermogravimetry (TG/DTG)}

Thermogravimetric analysis was carried out using a TGA 2050 (TA Instruments, New Castle, USA). Heating was performed in a platinum crucible in synthetic air flow 
(90 mL/min) at a rate of $10{ }^{\circ} \mathrm{C}$ minutes up to $800{ }^{\circ} \mathrm{C}$. The sample weight was in the range of $9-10 \mathrm{mg}$.

\section{Scanning Electron Microscopy (SEM)}

Samples of about $1 \mathrm{~cm}^{2}$ were coated with a thin layer of gold-palladium of $20 \mathrm{~nm}$ and the specimens were examined with a LEO 440 (LEO Electron Microscopy Ltd, Cambridge, England) with an accelerating voltage of $20 \mathrm{keV}$.

\section{Results and Discussion}

\section{Determination of the elastin content}

The elastin content determination is based on the assumed insolubility of elastin in $0.1 \mathrm{~mol} . \mathrm{L}^{-1} \mathrm{NaOH}^{[18]}$. Three independent determinations showed that the elastin content after alkaline hydrolysis and GA crosslinking does not change independent of the reaction time and GA concentration. Alkaline hydrolysis removes cells, in addition to other components present in the tissue, resulting in an acellular matrix composed of collagen and elastin ${ }^{[7]}$. So, it was possible to obtain the elastin and collagen mass percentage. The elastin average mass was $4.8 \pm 0.2 \%(\mathrm{w} / \mathrm{w})$ and collagen average mass was $95.2 \pm 0.2 \%(\mathrm{w} / \mathrm{w})$.

\section{In vitro trypsin degradation}

In vitro biodegradation test was performed to estimate the crosslinking extent. Trypsin acts in peptidic bonds (-CONH-) adjacent to the basic amino acids Lysine and arginine and has its activity restricted when these groups are blocked. In the case of collagen materials crosslinked with GA, inhibition occurs in Lys residues as a function of the Schiff base formation.

Figure 1 compares the biodegradation behavior of porcine skin and GA crosslinked matrices. After incubated in trypsin solution for 2 hours the 0GA matrix was $85 \%$ biodegraded. After GA crosslinking, the biodegradability enhanced and with the increase of GA concentration, there was a decrease of degradation. This can be observed comparing matrices crosslinked for 15 and 45 minutes.

The degradation percentage of matrices crosslinked with the same GA concentration, but with different reaction times (matrices 005GA15 and 005GA45) suggest there is a decreasing of the matrix degradation percentage with an increase of the reaction time and consequently, the matrix is more crosslinked.

The sequentially crosslinked matrix (85GA45S) showed a higher degradation percentage than the non-sequentially crosslinked matrix (85GA45). Therefore, the 85GA45S matrix is less crosslinked than the 85GA45 matrix. Matrices with different crosslinking degrees could be interesting in soft tissue reconstruction.

The higher resistance to degradation of the nonsequentially crosslinked matrix can be associated to the impermeable effect that occurs by the GA polymerized in the

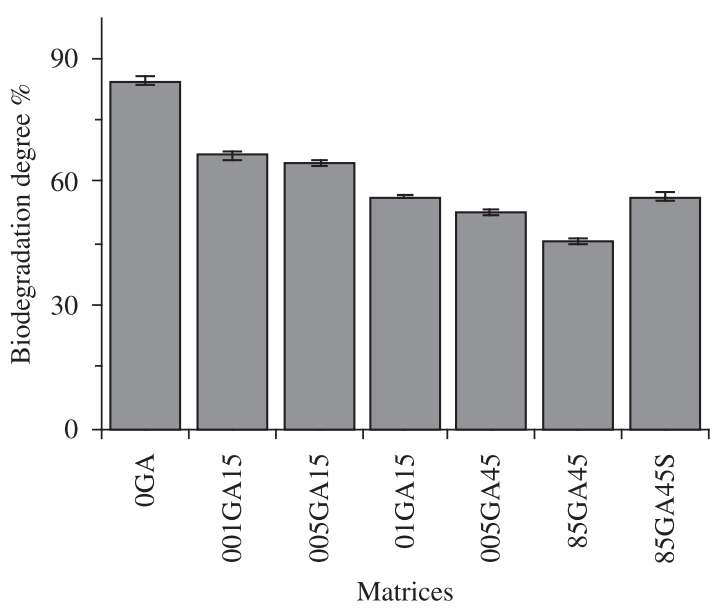

Figure 1. The biodegradation behavior ${ }^{\mathrm{a}}$ of porcine skin and GA crosslinked

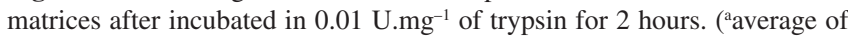
3 independent determinations).

matrix surface, preventing the enzyme from penetrating the tissue. Therefore, only non-crosslinked sites of the material surface will be exposed to enzymatic attacks.

This impermeable effect occurs when the matrix is crosslinked with a concentrated solution of $\mathrm{GA}^{[19]}$, which promotes a fast crosslinking of the tissue surface, generating a barrier that prevents or avoids the GA diffusion inside the tissue ${ }^{[20]}$. Thus, the inner regions of the collagen matrix remain without any type of crosslinking.

\section{Differential Scanning Calorimetry (DSC)}

To evaluate the effects of alkaline hydrolysis and GA crosslinking on the thermal stability on the collagen found in porcine skin, we performed DSC analysis. This assay is based on endothermic transitions as a measure of helicoidal structure stability of collagen molecules, related to the transition collagen $\rightarrow$ gelatin. When collagen is heated the matrix denatures at a specific temperature, resulting in the material's shrinkage to about one-third of its original length. In DSC curves, the denaturation transition appears as a baseline discontinuance, which is proportional to the heat capacity difference after and before denaturation. The transition temperature can be defined as either the onset or the maximum peak. In this work the inflection point was used to obtain the denaturation temperature (Td) values. The results of thermal denaturation temperature are summarized in Table 1 and Figure 2.

DSC curves shown in Figure 2 allowed the obtention of $\mathrm{Td}$ for all matrices, suggesting that the collagen present in porcine skin was not denatured during alkaline hydrolysis. As expected, uncrosslinked collagen matrix (0GA) showed the lowest denaturation temperature $\left(41.8{ }^{\circ} \mathrm{C}\right)$ among all samples. In contrast, crosslinked collagen matrices had higher temperatures. The results indicated that the difference in thermal stability of GA crosslinked matrices depend on the crosslinking process and revealed a tendency to increase with higher treatment time and GA concentration. 
Table 1. Denaturation temperature (Td) for collagen matrices obtained from DSC curves.

\begin{tabular}{cc}
\hline Matrices & Td $\left({ }^{\circ} \mathbf{C}\right)$ \\
\hline Porcine skin ${ }^{\mathrm{a}}$ & 66.8 \\
0GA & 41.8 \\
001GA15 & 44.1 \\
005GA15 & 49.0 \\
01GA15 & 50.5 \\
005GA45 & 55.9 \\
85GA45 & 56.2 \\
85GA45S & 53.2 \\
\hline
\end{tabular}

${ }^{a}$ matrix not submitted to alkaline hydrolysis.

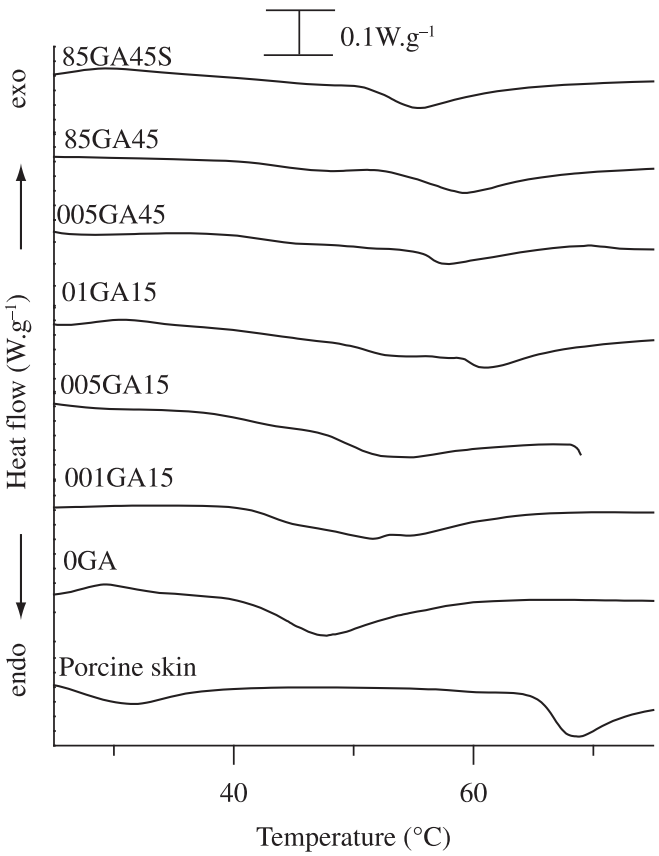

Figure 2. DSC curves of collagen matrices.

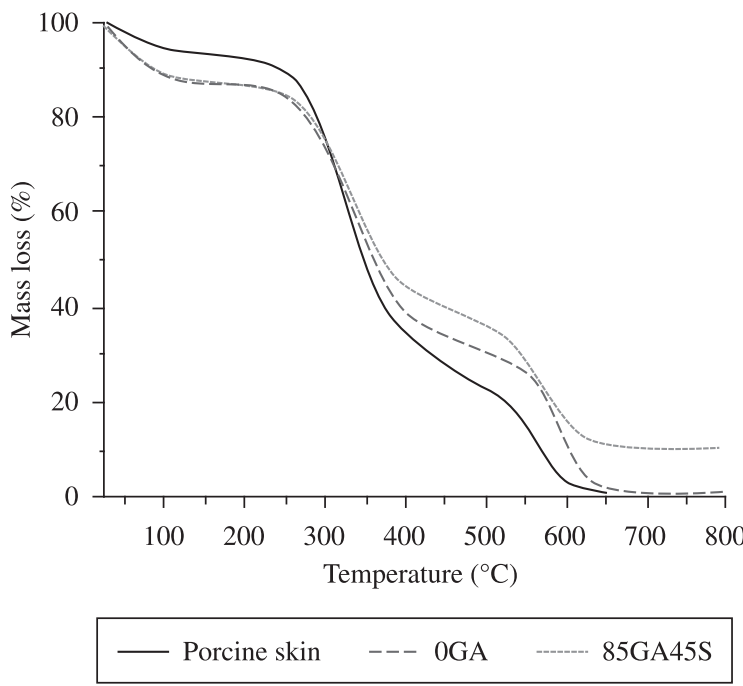

Figure 3. TG curves of collagen matrices.
The crosslinking can be attributed to the formation of Schiff base chemical bonds between GA and $\varepsilon$-amino groups of lysine and hydroxilysine present in the collagen matrix ${ }^{[16]}$.

The results obtained from DSC curves also indicated that after alkaline hydrolysis the $\mathrm{Td}$ value decreased approximately $25^{\circ} \mathrm{C}$. This effect can be associated to the disruption of natural crosslinking matrices and to the increase of negative charges in collagen with a disorganization of the microfibrillar structure ${ }^{[7]}$. This behavior was observed in other tissues as bovine pericardium submitted to the same alkaline hydrolysis.

Comparing $\mathrm{Td}$ values to the enzymatic degradation percentages, it was verified that the smaller the Td, the higher the enzymatic degradation percentage, as expected.

\section{Thermogravimetry (TG/DTG)}

Thermogravimetric curves and their respective first derivatives, which characterize thermal destruction of the matrices submitted to alkaline hydrolysis and GA crosslinking (85GA45S), are shown in Figures 3 and 4. All crosslinked collagen matrices showed the same behavior as that observed for the 85GA45S matrix.

TG/DTG results (Table 2) showed differences in water loss (between 25 and $200{ }^{\circ} \mathrm{C}$ ), suggesting that after the alkaline

Table 2. Mass loss (\%) of collagen matrices obtained by TGA/DTGA.

\begin{tabular}{lccc}
\hline \multicolumn{1}{c}{ Matrices } & \multicolumn{3}{c}{ Mass loss $(\boldsymbol{\%})$} \\
\cline { 2 - 4 } & $\mathbf{2 5 - 2 0 0}{ }^{\circ} \mathbf{C}$ & $\mathbf{2 0 0 - 4 8 0}{ }^{\circ} \mathbf{C}$ & $\mathbf{4 8 0 - 8 0 0}^{\circ} \mathbf{C}$ \\
\hline Porcine skin $^{\mathrm{a}}$ & 7.8 & 68.4 & 23.5 \\
0GA & 10.3 & 70.0 & 18.5 \\
001GA15 & 14.5 & 47.8 & 29.7 \\
005GA15 & 14.8 & 49.5 & 31.5 \\
01GA15 & 14.8 & 48.3 & 32.8 \\
005GA45 & 14.6 & 45.8 & 29.6 \\
85GA45 & 10.0 & 61.4 & 24.9 \\
85GA45S & 13.3 & 48.7 & 27.8 \\
\hline
\end{tabular}

${ }^{a}$ matrix not submitted to alkaline hydrolysis.

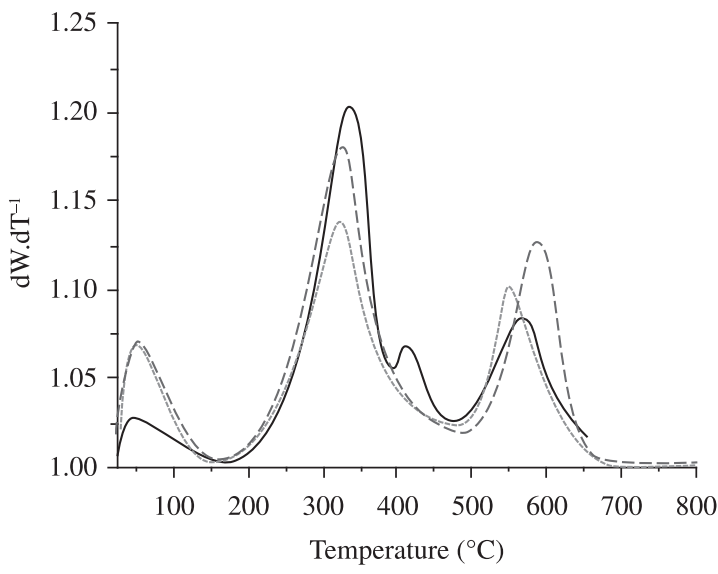

$$
\text { — Porcine skin --- 0GA }----85 \mathrm{GA} 45 \mathrm{~S}
$$

Figure 4. DTG curves of collagen matrices. 
hydrolysis there was a water content increase. This increase occurs due to the alkaline hydrolysis of carboxyamides groups of Asn and Gln residues that increase the negative charges (carboxyl groups) $)^{[7]}$, resulting in an increase of solvation effect in the collagen and then, a higher quantity of absorbed water. After the GA crosslinking reaction, this water quantity increased further, due to the increase of negative charge density through the GA reaction with Lys and Hyl $\varepsilon$-amino groups of collagen matrix to form chemical bonds type Schiff bases $(-\mathrm{CH}=\mathrm{N})^{[16]}$.

Comparing the differences in water loss between the 85GA45 and 85GA45S matrices, it can be observed that

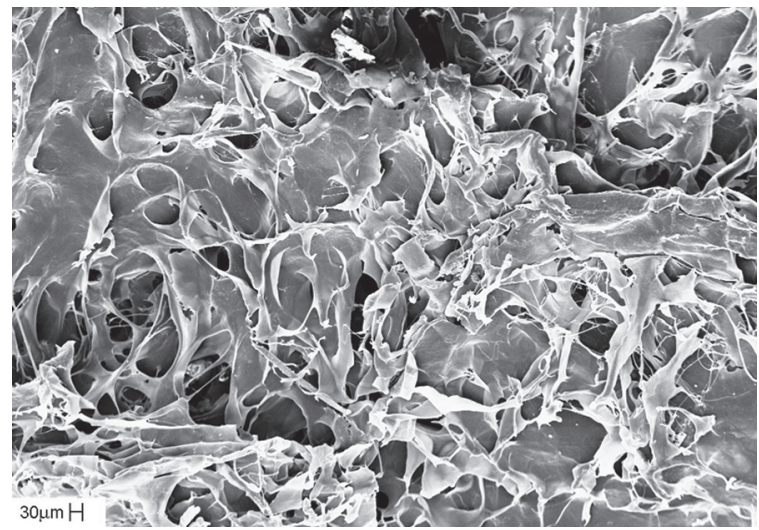

(a)

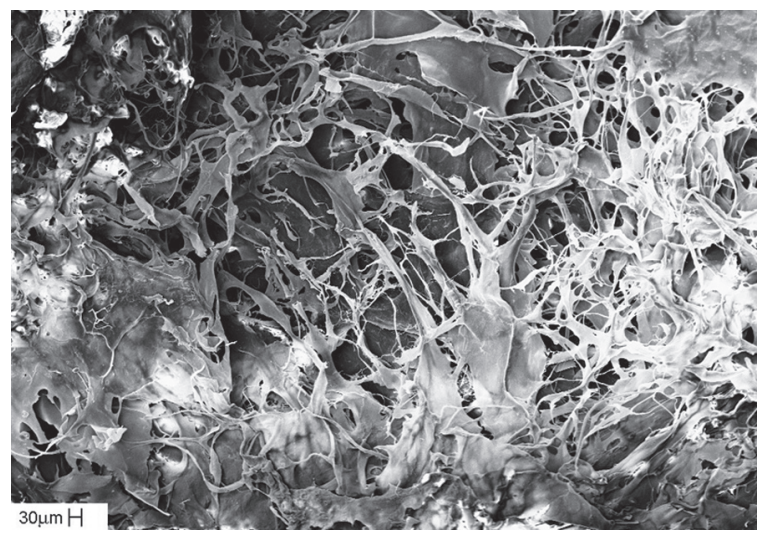

(c)

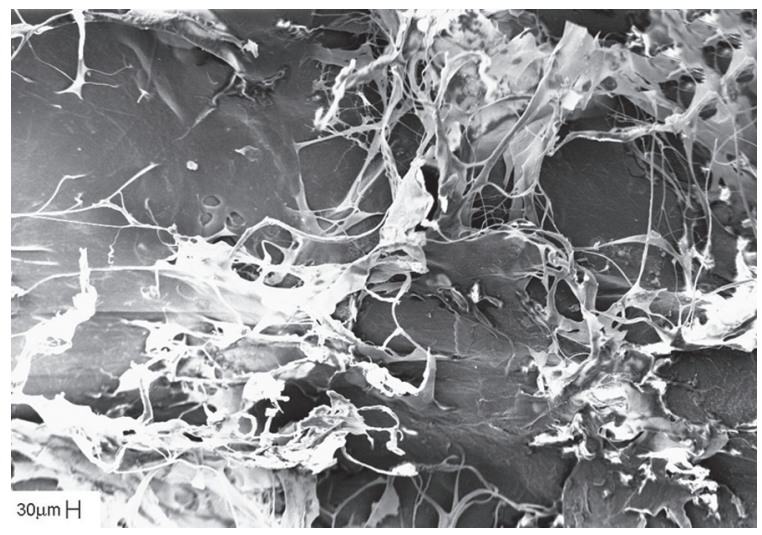

(e) the matrix crosslinked non-sequentially (85GA45) shows a smaller water quantity than the matrix sequentially crosslinked (85GA45S), suggesting that $85 \mathrm{GA} 45$ is more impermeable.

In the temperature range of $200-480{ }^{\circ} \mathrm{C}$, collagen and elastin decomposition can be observed in the hydrolysed and crosslinked matrices. In crosslinked matrices, the mass loss percentage was smaller due to the formation of chemical bonds type Schiff bases rendering the collagen thermally more stable ${ }^{[16]}$. Accordingly, part of the collagen was degraded in this temperature range and the remaining was carbonized.

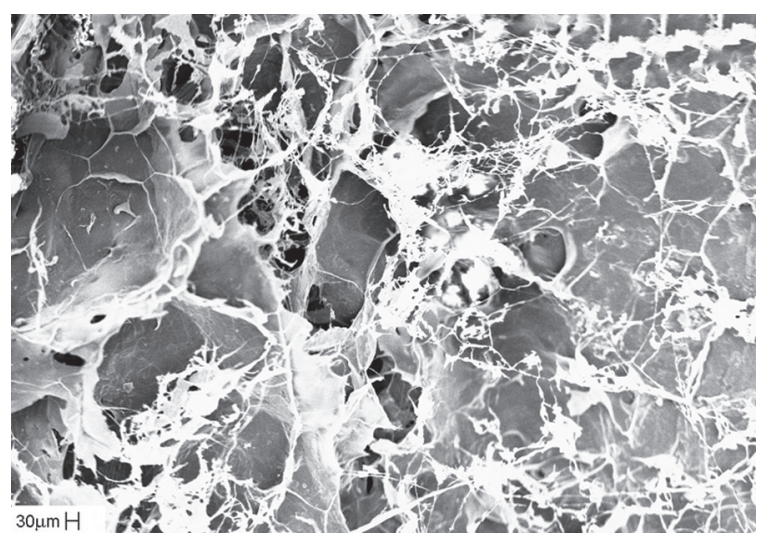

(b)

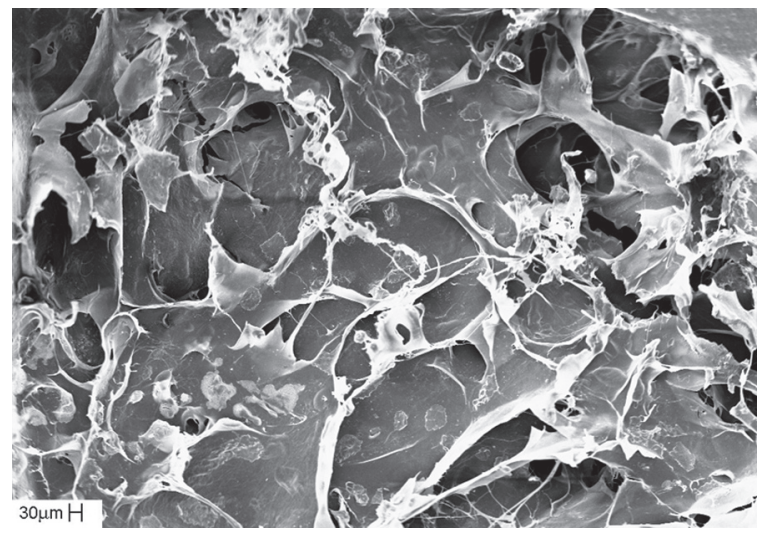

(d)

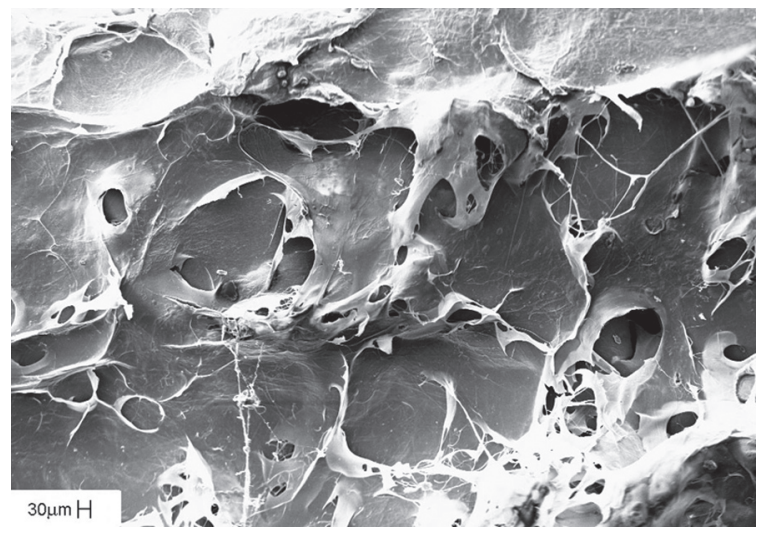

(f)

Figure 5. SEM images for collagen matrices from different treatments: a) 0GA; b) 001GA15; c) 005GA15; d) 005GA45; e) 85GA45; f) 85GA45S. (200×). 
On the other hand, in the range of $480-800{ }^{\circ} \mathrm{C}$, the crosslinked matrices had a higher loss of mass in relation to the non-crosslinked ones. Therefore, part of the protein was carbonized as it is more resistant to thermal degradation.

Our results have shown that alkaline hydrolysis and GA treatment cause an increase in the water content of collagen matrices.

\section{Scanning Electron Microscopy (SEM)}

Figure 5 illustrates the SEM examination of superficial morphologies of collagen matrices after alkaline hydrolysis and GA crosslinking. Morphological variations can be seen in the matrix surface after GA crosslinking. It is expected that different cross-linking procedures can modify the spatial organization of collagen fibrils and their surface topography ${ }^{[21]}$.

The 0GA micrograph (Figure 5a) as a reference, exhibited a dense porous structure. After GA crosslinking there were some morphological changes, as shown in Figure $5 \mathrm{~b}$ and c. They look less porous than the non-crosslinked matrix, indicating that GA crosslinking increases the aggregation of collagen fibers, resulting in a more compact structure. The effects of the increasing GA concentration and/or reaction time on the matrices' structure were observed (Figure 5d and f).

Apparently, there were no high alterations and the structural pattern of the collagen fibers still remained in all matrices; hence, the natural structure of porcine skin was preserved after treatments and the modifications of collagen fibers might be minimal.

\section{Conclusions}

Alkaline hydrolysis and GA crosslinking did not induce collagen denaturation and did not remove the elastin found in the matrices.

The crosslinking reaction with GA increases $\mathrm{Td}$ values and decreases the enzymatic degradation. The crosslinking degree is higher with the increase of GA concentration or reaction time. In addition, the non-sequentially crosslinked matrix was more stable in relation to the one crosslinked sequentially.

Thus, using differentiated crosslinking reactions it is possible to obtain matrices with different biological stabilities, increasing the range of applications in the field of soft tissue reconstruction.

\section{Acknowledgments}

The authors thank Ezer Biazin and Glauco D. Broch for technical support and Carlos A. S. Bento for SEM analysis. Fabiana T. Rodrigues has a CAPES fellowship (Coordenação de Aperfeiçoamento de Pessoal de Nível superior) at the postgraduation program at the Instituto de Química de São Carlos, Universidade de São Paulo (USP), São Carlos, SP, Brazil.

\section{References}

1. Middelkoop, E.; Van Den Bogaerdt, A. J.; Lame, E. N.; Hoekstra, M. J.; Brandsma, K. \& Ulrich, M. M. W. - Biomaterials, 25, p. 1559 (2004).

2. Hoyama, E.; Schellini, S. A.; Pellizon, C. H.; Marques, M. E. A.; Padovani, C. R. \& Rossa, R. - An. Bras. Dermatol., 80, p. 369 (2005).

3. Pereira, C.; Gold, W. \& Herndon, D. - J. Biomater. Appl., 22, p. 101 (2007).

4. Shaikh, F. M.; Giri, S. K.; Durrani, S.; Waldron, D. \& Grace, P.A. - World J. Surg., 31, p. 1966 (2007).

5. Ruszczak, Z. - Adv. Drug Del. Rev., 55, p. 1595 (2003).

6. Prasertsung, I.; Kanokpanont, S.; Bunaprasert, T.; Thanakit, V. \& Damrongsakkul, S. - J. Biomed. Mater. Res. B Appl. Biomater., 85, p. 210 (2008).

7. Bet, M. R.; Goissis, G. \& Lacerda, C.A. - Biomacromolecules, 2, p. 1074 (2001).

8. Goissis, G.; Marcantonio Junior, E.; Marcantônio, R. A. C.; Lia, R. C. C.; Cancian, D. C. J. \& Carvalho, W. M. - Biomaterials, 20, p. 27 (1999).

9. Rocha, L. B.; Goissis, G. \& Rossi, M. A. - Biomaterials, 23, p. 449 (2002).

10. Badylak, S. F.; Freytes, D. O. \& Gilbert, T. W. - Acta Biomater., 5, p. 1 (2009).

11. Forti, F. L.; Goissis, G. \& Plepis, A. M. G. - J. Biomater. Appl., 20, p. 267 (2006).

12. Goissis, G.; Braile, D. M. \& Giglioti, A. F. - Polímeros, 9, p. 92 (1999).

13. Jarman-Smith, M. L.; Bodamyali, T.; Stevens, C.; Howell, J. A.; Horrocks, M. \& Chaudhuri, J. B. - J. Mater. Sci. Mater. Med., 15, p. 925 (2004).

14. Liang, H. C.; Chang, Y.; Hsu, C. K.; Lee, M. H. \& Sung, H. W. - Biomaterials, 25, p. 3541 (2004).

15. Sheu, M. T.; Huang, J. C.; Yeh, G. C. \& Ho, H. O. - Biomaterials, 22, p. 1713 (2001).

16. Fathima, N. N.; Madhan, B.; Rao, J. R.; Nair, B. U. \& Ramasami, T. - Int. J. Biol. Macromol., 34, p. 241 (2004).

17. Bet, M. R.; Goissis, G.; Vargas, S. \& Selistre de Araújo, H. S. - Biomaterials, 24, p. 131 (2003).

18. Cross, H. R.; Smith, G. C. \& Carpenter, Z. L. - J. Agric. Food Chem., 21, p. 716 (1973).

19. Newton, D.; Mahajan, R.; Ayres, C.; Bowman, J. R., Bowlin, G. L. \& Simpson, D. G. - Acta Biomater., 5, p. 518 (2009).

20. Saito, H.; Murabayashi, S.; Mitamura, Y. \& Taguchi, T. - J. Mater. Sci. Mater. Med., 19, p. 1297 (2008).

21. Langdon, S. E.; Chernecky, R.; Pereira, C. A.; Abdulla, D. \& Lee, J. M. - Biomaterials, 20, p. 137 (1999).

Enviado: 04/06/09

Aceito: $23 / 12 / 09$

DOI: 10.1590/S0104-14282010005000013 